\title{
Limited usefulness of serum carcinoembryonic antigen and carbohydrate antigen 19-9 on the preoperative diagnosis of extrahepatic bile duct cancer
}

Hyeong Seok KIM, Youngmin HAN, Jae Seung KANG, Yoon Hyung KANG, Mirang LEE, Hee Ju SOHN, Hongbeom KIM, Wooil KWON, Jin-Young JANG*

Department of Surgery and Cancer Research Institute, Seoul National University College of Medicine, Seoul, Korea

Introduction: Littles is known about the role of serum carcinoembryonic antigen (CEA) and carbohydrate antigen (CA) 19-9 on the detection of extrahepatic bile duct cancer (EBDC). This study evaluated the diagnostic accuracy of these biomarkers.

Methods: A total of 710 patients who underwent surgery for EBDC at a tertiary center between 1995 and 2018 were included, with 23 patients excluded owing to concurrent pancreatobiliary diseases, which could affect serum CEA or CA19-9. For a control group, 2,310 patients who underwent cholecystectomy for benign gallbladder diseases during the same period were used. Diagnostic accuracy was evaluated using sensitivity, specificity, and area under the receiver operating characteristics curve (AUC).

Results: After excluding patients, 687 qualifying patients consisted of 488 men and 199 women with a mean age of 65.8 years. Median serum levels of CEA and CA19-9 were $1.8 \mathrm{ng} / \mathrm{mL}$ and $47.0 \mathrm{U} / \mathrm{mL}$, respectively. CEA (cut-off: $5.0 \mathrm{ng} / \mathrm{mL}$ ) showed AUC of 0.541 , sensitivity of $9.0 \%$, and specificity of $99.2 \%$, and CA19-9 (cut-off: $37 \mathrm{U} / \mathrm{mL}$ ) had AUC of 0.753 , sensitivity of $56.2 \%$, and specificity of $94.5 \%$. According to AJCC stage, early T stage (stage 0-2) had lower sensitivity in CA19-9 (47.0\% vs. 64.9\%) than advanced T stage (stage 3 and 4). In addition, N0 stage showed lower sensitivity (50.1\% vs. 68.8\%) than lymph node metastasis.

Conclusions: Serum CEA and CA19-9 have limited usefulness for the diagnosis of EBDC, particularly in early detection, because of their quite low sensitivity. A novel biomarker for the diagnosis of cancer will also be needed in the future. 